\section{Occurrence of Hashimoto thyroiditis among the first- and second-degree relatives of systemic lupus erythematosus patients with Hashimoto thyroiditis}

\author{
L. Carli, C. Tani, S. Vagnani, F. Querci, C. Baldini, R. Talarico, \\ A. Della Rossa, S. Bombardieri, M. Mosca \\ Rheumatology Unit, University of Pisa, Italy
}

Sirs,

A number of studies have suggested that thyroid disorders, including Hashimoto thyroiditis (HT), are more frequent in SLE than in general population, with a reported prevalence ranging from 3.9 to $24 \%(1,2)$. Since a genetic background has been described for systemic autoimmune diseases as well as for autoimmune thyroid diseases (AITD), and familial aggregation of systemic autoimmune diseases and AITD has been described (3-6), we have hypothesized that first and second degree relatives of systemic lupus erythematosus (SLE) patients with HT might have an increased risk of developing this condition.

The clinical charts of patients with SLE were examined, to identify which subjects suffered from HT, based on the presence of positive thyroid antibodies and ultrasound assessment suggestive of an inflammatory disease.

Patients were asked about the presence of thyroid disorders in their first- and seconddegree relatives, using a structured questionnaire, designed ad hoc for this study (Appendix).

We considered valid the presence of HT when the patient reported specifically that diagnosis.

The clinical charts of 401 SLE patients (371 female, 30 male) were examined. Sixty-eight (17\%), 65 women and 3 men had a diagnosis of HT, 12 were lost to follow-up. Of the remaining 56, $28(50 \%)$ had at least one first- or second-degree relative with a thyroid disease. A total of 36 relatives (30 women, 6 men) with a thyroid dysfunction were observed. Eight were affected by HT, 8 by MNG, while the diagnosis was unclear in 20 relatives, with 19 cases $(53 \%$ of the total number of relatives) with hypothyroidism and 1 case of hyperthyroidism. Two relatives were reported to have had a history of papillary carcinoma.

Hashimoto thyroiditis is a common cause of hypothyroidism, with a reported prevalence of $1-4 \%$ in the general population $(6$, 7).

A familial aggregation of autoimmune thyroiditis has been described and the positivity of anti-TPO antibodies has been reported in $27-34 \%$ of the relatives of patients with autoimmune thyroiditis. In addition a parental history of hypo or hyperthyroidism has been reported in about $20 \%$ of mothers of subjects with HT (6).

Various studies have shown an increased prevalence of HT in first and second degree relatives of SLE patients $(3,4)$; aim of the present study was to test the hypothesis as to whether relatives of patients with SLE and HT could be at increased risk of developing HT and should therefore be screened for such condition.

A diagnosis of HT was reported in $22 \%$ of first and second-degree relatives of SLE patients with HT. However, these data may underestimate the real prevalence of HT as it could be reasonable to consider the generic hypothyroidism reported in 20 relatives as possible cases of HT.

Secondly, relatives were not re-evaluated and this could determine an additional
Corresponding author: Marta Mosca Rheumatology Unit University of Pisa Via Roma, 67 - 56126 Pisa, Italy E-mail: marta.mosca@med.unipi.it 
underestimation of the real prevalence of thyroid disorders in the families of SLE patients with HT.

To our knowledge this is the first study assessing the risk of HT among relatives of SLE patients with HT. Based on these results, it might be interesting to perform additional studies on larger populations, with a direct evaluation of relatives; this could help in further clarifying if relatives of SLE patients with HT should be counseled to evaluate the presence of autoimmune thyroid diseases.

\section{REFERENCES}

1. Pyne D, Isenberg DA. Autoimmune thyroid disease in systemic lupus erythematosus. Ann Rheum Dis. 2002; 61: 70-2.

2. Antonelli A, Fallahi P, Mosca M, Ferrari SM, Ruffilli I, Corti A, et al. Prevalence of thyroid dysfunctions in systemic lupus erythematosus. Metabolism. 2010; 59: 896-900.
3. Mosca M, Carli L, d'Ascanio A, Tani C, Talarico $\mathrm{R}$, Baldini $\mathrm{C}$, et al. Occurrence of organspecific and systemic autoimmune diseases among the first- and second-degree relatives of Caucasian patients with connective tissue diseases: report of data obtained through direct patients interviews. Clin Rheumatol. 2008; 27: 1045-48.

4. Alarçon-Segovia D, Alarçon-Riquelme ME, Cardiel MH, Caeiro F, Massardo L, Villa AR, et al. Familial aggregation of systemic lupus erythematosus, rheumatoid arthritis and other autoimmune diseases in 1177 lupus patients from the GLADEL cohort. Arthritis Rheum. 2005; 52: 1138-47.

5. Boelaert K, Newby PR, Simmonds MJ, Holder RL, Carr-Smith JD, Heward JM, et al. Prevalence and relative risk of other autoimmune diseases in subjects with autoimmune thyroid disease. Am J Med. 2010; 123: 183.e1-e9.

6. Marwaha RK, Sen S, Tandon N, Sahoo M, Walia RP, Singh S, et al. Familial aggregation of autoimmune thyroiditis in first-degree relatives of patients with juvenile autoimmune thyroid disease. Thyroid. 2003; 13: 297-300.

7. Roberts CGP, Ladenson PW. Hypothyroidism. Lancet. 20004; 363: 793-803. 